\title{
Evidence for the formation of a Mott state in potassium-intercalated pentacene
}

\author{
Monica F. Craciun, ${ }^{1,2}$ Gianluca Giovannetti, ${ }^{3,4}$ Sven Rogge, ${ }^{1}$ Geert Brocks, ${ }^{4}$ \\ Alberto F. Morpurgo, ${ }^{1,5}$ and Jeroen van den Brink ${ }^{3,6,7}$ \\ ${ }^{1}$ Kavli Institute of Nanoscience, Delft University of Technology, Lorentzweg 1, 2628 CJ Delft, The Netherlands \\ ${ }^{2}$ Department of Applied Physics, The University of Tokyo, Tokyo 113-8656, Japan \\ ${ }^{3}$ Institute Lorentz for Theoretical Physics, Leiden University, 2300 RA Leiden, The Netherlands \\ ${ }^{4}$ Faculty of Science and Technology and MESA + Institute for Nanotechnology, University of Twente, 7500 AE Enschede, \\ The Netherlands \\ ${ }^{5}$ DPMC and GAP, University of Geneva, quai Ernest-Ansermet 24, CH-1211 Geneva 4, Switzerland \\ ${ }^{6}$ Institute for Molecules and Materials, Radboud University, 6500 GL Nijmegen, The Netherlands \\ ${ }^{7}$ Stanford Institute for Materials and Energy Sciences, Stanford University and SLAC National Accelerator Laboratory, Menlo Park, \\ California 94025, USA
}

(Received 5 November 2008; revised manuscript received 9 January 2009; published 24 March 2009)

\begin{abstract}
We investigate electronic transport through pentacene thin films intercalated with potassium. From temperature-dependent conductivity measurements we find that potassium-intercalated pentacene shows metallic behavior in a broad range of potassium concentrations. Surprisingly, the conductivity exhibits a reentrance into an insulating state when the potassium concentration is increased past one atom per molecule. We analyze our observations theoretically by means of electronic structure calculations, and we conclude that the phenomenon originates from a Mott metal-insulator transition, driven by electron-electron interactions.
\end{abstract}

DOI: 10.1103/PhysRevB.79.125116

PACS number(s): 73.61.Ph, 71.20.Tx, 71.30. $+\mathrm{h}$

\section{INTRODUCTION}

Pentacene (PEN) is a conjugated molecule very well known in the field of plastic electronics for its use in highmobility organic thin-film transistors. ${ }^{1,2}$ Plastic electronic applications rely on the fact that at low density of charge carriers pentacene films effectively behave as weakly doped semiconductors..$^{3-5}$ In this regime, which is studied extensively, the interactions between charge carriers can be neglected. However, the opposite regime of high carrier density has remained virtually unexplored. Since pentacene forms a molecular solid with narrow bandwidth it can be expected that at high density the simple assumptions of independent electron-band theories break down and the electronic correlations determine the electronic properties of the material, ${ }^{6}$ as it happens in other molecular systems. The origin of correlated behavior in these systems is the competition between the energy gained by delocalizing the $\pi$ electrons (given by the electronic bandwidth) and the Coulomb repulsion between two carriers on the same molecule. If the repulsion energy is larger than the one gained on delocalization, then the electrons become localized and a Mott metal-insulator transition takes place. Among the most studied molecular systems in the high carrier density regime are the intercalated $\mathrm{C}_{60}$ crystals $^{7}$ and the organic charge-transfer salts, where the electronic interactions lead to the appearance of highly correlated magnetic ground states and unconventional superconductivity. ${ }^{8,9}$

Our goals are to investigate pentacene compounds at high carrier density - of the order of one carrier per moleculeand to show that electron correlation effects are crucial to understand the resulting electronic properties. To this end, we have studied electronic transport through high-quality pentacene thin films similar to those used for the fabrication of field-effect transistors. In order to reach high densities of charge carriers we intercalate the pentacene films with potassium atoms to form $\mathrm{K}_{x} \mathrm{PEN}$. Several past experimental studies have addressed the possibility to chemically dope pentacene thin films through the inclusion of alkali atoms and iodine. For all these compounds the structural investigations have shown that large concentrations of atoms (up to three iodine atoms per pentacene molecule) can intercalate in between the planes of the pentacene molecular films. Similar to the case of intercalated $\mathrm{C}_{60},{ }^{10}$ the alkali atoms donate their electrons to the lowest unoccupied molecular orbital (LUMO) of the pentacene molecules, whereas the iodine donate holes to the highest occupied molecular orbital (HOMO), enabling the control of the conductivity of the films. Earlier studies ${ }^{11-18}$ indicate that upon iodine or rubidium intercalation the conductivity of pentacene films can become large (in the order of $100 \mathrm{~S} / \mathrm{cm}$ ) and exhibit a metallic temperature dependence. The experiments so far, however, have not led to an understanding of the doping dependence of the conductivity (e.g., how many electrons can be transferred upon intercalation) or of the microscopic nature of electrical conduction of doped pentacene in the high carrier density regime.

In this paper we present the experimental investigation of the evolution of the temperature-dependent conductivity of potassium-intercalated pentacene thin films, with increasing their doping concentration. We find that, upon $\mathrm{K}$ intercalation, PEN films become metallic in a broad range of doping concentrations, up to $\mathrm{K}_{1} \mathrm{PEN}$, after which the conductivity re-enters an insulating state. Our experiments also show that the structural disorder of PEN films plays an important role on the transport properties of $\mathrm{K}_{x} \mathrm{PEN}$, as films of poor structural quality do not exhibit metallic behavior. The analysis of our data shows that at high carrier density the conductivity of 
$\mathrm{K}_{1} \mathrm{PEN}$ cannot be described in terms of independent electrons filling the molecular band originating from the LUMO. Rather, our observations are consistent with the formation of a Mott insulating state, driven by electron-electron interactions, as we show theoretically by calculating the electronic structure of $\mathrm{K}_{1} \mathrm{PEN}$.

\section{PREPARATION OF $\mathrm{K}_{x}$ PEN FILMS}

Our choice of working with pentacene thin films (as opposed to single crystals) is motivated by both the relevance for applications - control of doping in organic semiconductors is important for future plastic electronic devices-and by the difficulty to grow crystals of alkali-intercalated pentacene, which has so far impeded this sort of investigations. All technical steps of our experimental investigations including the deposition of pentacene films, potassium intercalation, and temperature-dependent transport measurements have been carried out in ultrahigh vacuum (UHV) $\left(10^{-11} \mathrm{mbar}\right)$ in a fashion that is similar to our previous studies of intercalated phthalocyanine films. ${ }^{19}$ The use of UHV prevents the occurrence of degradation of the doped films over a period of days.

As with the phthalocyanines, the PEN films $(\sim 25 \mathrm{~nm}$ thick) were thermally evaporated from a Knudsen cell onto a silicon substrate kept at room temperature. In order to minimize the parallel conduction through the silicon, we use a high resistive silicon-on-insulator (SOI) wafer as substrate. The SOI wafer consists of $2 \mu \mathrm{m}$ silicon top layer electrically insulated by $1-\mu \mathrm{m}$-thick $\mathrm{SiO}_{2}$ layer from the silicon substrate. ${ }^{19} \mathrm{Ti} / \mathrm{Au}$ electrodes $(10 \mathrm{~nm} \mathrm{Ti}$ and $50 \mathrm{~nm} \mathrm{Au})$ were deposited ex situ on the SOI substrates [see Fig. 1(b)]. After the deposition of the electrodes and prior to loading the substrate into the UHV system, a hydrogen-terminated Si surface was prepared by dipping the SOI surface in a hydrofluoric acid solution and rinsing in de-ionized water. The use of such a H-terminated silicon surface proved necessary to achieve sufficient quality in film morphology, as we will discuss in Sec. III in more detail.

Special care was taken to chemically purify pentacene prior to the film deposition. As-purchased pentacene powder was purified by means of physical vapor deposition in a temperature gradient in the presence of a stream of argon gas as described in Ref. 20. After this step, the pentacene powder was loaded in the Knudsen cell in the UHV system and was further purified by heating it at a temperature just below the sublimation temperature for several days. The film thickness was determined by calibrating the pentacene deposition rate ex situ using an atomic force microscope (AFM).

Potassium doping was achieved by exposing the films to a constant flux of $\mathrm{K}$ atoms generated by a current-heated getter source. The source was calibrated and the potassium concentration determined by means of an elemental analysis performed on PEN films doped at several doping levels using ex situ Rutherford backscattering (RBS). As shown in the top inset of Fig. 1 the ratio of $\mathrm{K}$ atoms to PEN molecules, $N_{\mathrm{K}} / N_{\mathrm{PEN}}$, increases linearly with increasing the doping time, as expected. Deviations from linearity-approximately $10 \%-20 \%$ - are due to inhomogeneity of the potassium concentration.

\section{TRANSPORT PROPERTIES OF $\mathrm{K}_{x}$ PEN}

\section{A. Electronic transport through high structural-quality $K_{x}$ PEN films}

The conductance of $\mathrm{K}_{x} \mathrm{PEN}$ films is measured in situ in a two terminal measurement configuration with a contact separation of approximately $175 \mu \mathrm{m}$ [see Fig. 1(b)]. The dependence of the conductivity on the potassium concentration, hereafter referred to as the "doping curve," is determined for different PEN films as a function of the ratio of $\mathrm{K}$ atoms to PEN molecules. The doping curves for different samples are very similar, as shown in Fig. 1(a). Upon doping, the conductivity initially increases rapidly up to a value of $\sigma$ $\sim 100 \mathrm{~S} / \mathrm{cm}$ - in the same range as the conductivity of metallic $\mathrm{K}_{3} \mathrm{C}_{60}{ }^{21}$ Upon doping further, the conductivity continues to increase more slowly, reaches a maximum at a concentration of $1 \mathrm{~K} / \mathrm{PEN}$, and then drops sharply back to the value of the undoped PEN film. All of the more than 40 films that we have investigated exhibit a similar behavior.

The observed suppression of the conductivity of pentacene films at high doping (for potassium concentrations higher than $1 \mathrm{~K} / \mathrm{PEN}$ ) allows us to exclude the possibility that the conduction of the intercalated films observed in the experiments is due to an experimental artifact, for instance, the formation of a potassium layer on top of the pentacene film. In fact, at doping higher than $1 \mathrm{~K} / \mathrm{PEN}$ the measured conductance, and its temperature dependence, is essentially identical to what is measured for pristine films.

To understand the nature of conduction of pentacene films at high carrier density we measured the temperature dependence of the conductivity for different values of potassium concentration [see Fig. 2(a)]. Pristine PEN films have a very low conductivity and the measured conductance of undoped films is dominated by transport through the substrate's $2-\mu \mathrm{m}$-thick $\mathrm{Si}$ top layer. The measured conductivity decreases rapidly with lowering temperature, as expected, confirming that undoped $(x=0)$ pentacene films are insulating. On the contrary, in the highly conductive state-for $x$ between 0.1 and 1 - the conductance of the films remains high down to the lowest temperature reached in the experiments $(\sim 5 \mathrm{~K})$, indicating a metallic state. When the potassium concentration is increased beyond approximately $1 \mathrm{~K} / \mathrm{PEN}$, the conductivity again decreases rapidly with lowering temperature, indicating a re-entrance into an insulating state. The metallic and insulating nature of pentacene thin films at different potassium concentrations is confirmed by measurements of volt-amperometric characteristics ( $I-V$ curves) at 5 $\mathrm{K}$. For $x$ between 0.1 and 1 the films exhibit linear $I-V$ characteristics, as expected for a metal [Fig. 2(b)]. On the contrary, in the highly doped regime (for $x>1$ ), the insulating state manifests itself in strongly nonlinear $I-V$ curves and virtually no current flowing at low bias [Fig. 2(c)]. Therefore, the data clearly show that pentacene films undergo a metal-insulator transition as the density of potassium is increased past one atom per molecule. Since in the overdoped regime the conduction occurs through the Si layer of the SOI substrate, it is not possible to gain specific information about the properties of the insulating KPEN films-for instance, to determine the electronic gap from measurements of the activation energy of the conductivity-by studying dc transport on our samples. 

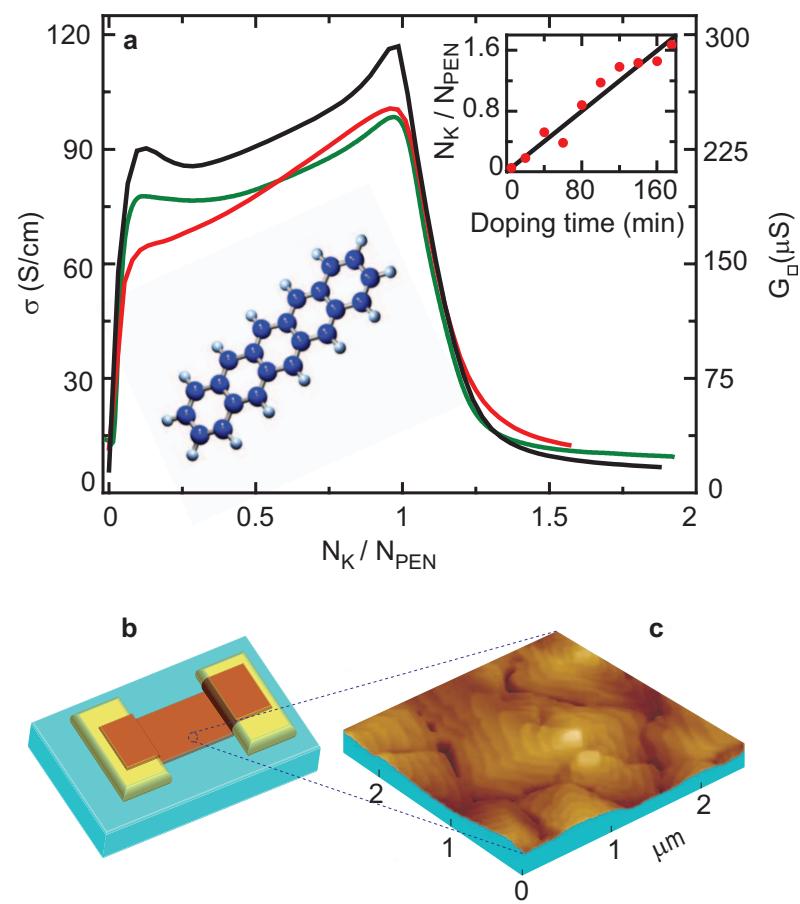

FIG. 1. (Color) (a) Conductivity $\sigma$ and square conductance $G_{\square}$ of three different K-doped PEN films as a function of the ratio $N_{\mathrm{K}} / N_{\mathrm{PEN}}$; under the curves a pentacene molecule. Inset: $N_{\mathrm{K}} / N_{\mathrm{PEN}}$ as a function of doping time. Schematic view (b) of our setup and (c) atomic force microscopy image of a high-quality undoped PEN film showing large crystalline grains.

\section{B. Effect of structural disorder on the transport properties of $\mathrm{K}_{x} \mathrm{PEN}$}

The high structural quality of the films proves to be the essential ingredient necessary to obtain $\mathrm{K}_{x} \mathrm{PEN}$ films which exhibit metallic conductivity. We find that the quality of pentacene thin films is highly sensitive to the choice of the substrate material and sufficient quality can be achieved by using a hydrogen-terminated $\mathrm{Si}$ surface. To illustrate this important technical point, we show here that the structural quality of films deposited on $\mathrm{SiO}_{2}$ surface has a very large impact on their electronic transport, with low quality resulting in considerably poorer electrical properties.

Figure 3(a) shows the doping curve of PEN films deposited onto $300 \mathrm{~nm} \mathrm{SiO}_{2}$ that was thermally grown on a $\mathrm{Si}$ substrate. For these films, the maximum conductivity that we measured experimentally is several orders of magnitude lower than the conductivity measured for films deposited on a $\mathrm{Si}$ surface. In addition, (on $\mathrm{SiO}_{2}$ ) the conductivity was always observed to decrease rapidly with lowering temperature, i.e., the potassium-intercalated films are always insulating [see Fig. 3(b)]. Both the magnitude and the temperature dependence of the conductivity that we measured on $\mathrm{SiO}_{2}$ substrates are comparable to results obtained in earlier work reported in the literature.

We attribute the difference in the electrical behavior observed for films deposited on $\mathrm{Si}$ and $\mathrm{SiO}_{2}$ substrates to the difference in film morphology, which we have analyzed using an atomic force microscope. Figure 3 shows AFM images of two pentacene films of similar thickness deposited on
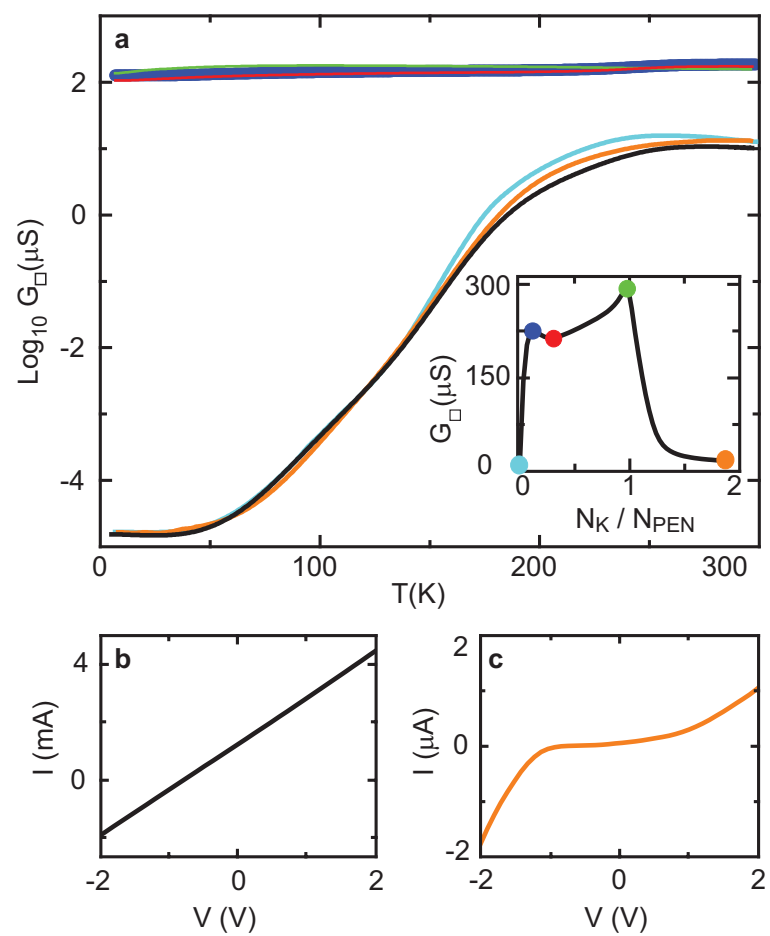

FIG. 2. (Color) Temperature dependence of the conductance of potassium-intercalated pentacene films. (a) The colored dots in the inset of (a) indicate the doping level at which the temperaturedependent conductivity measurements with corresponding color were performed. In black the temperature-dependent conductivity of the Si substrate is shown. The low temperature $(5 \mathrm{~K}) I-V$ characteristics of $\mathrm{K}_{x} \mathrm{PEN}$ in the (b) conducting and (c) highly doped insulating states.

the $\mathrm{SiO}_{2}$ surface [Fig. 3(c)] and on the hydrogen-terminated $\mathrm{Si}$ [Fig. 3(d)]. It is apparent that very different morphologies are observed for the two substrates. PEN films deposited on Si surfaces exhibit large crystalline grains with a common relative orientation and only relatively small fluctuations in height. On $\mathrm{SiO}_{2}$, on the contrary, the grains are much smaller, randomly oriented, and they exhibit much larger height fluctuations.

This conclusion is consistent with past studies ${ }^{22}$ showing that the growth and morphology of pentacene films are strongly influenced by the substrate surface. Specifically, for pentacene films grown on $\mathrm{SiO}_{2}$, a high density of nucleation centers was observed, leading to the growth of small islands and to a high concentration of grain boundaries. On the hydrogen-terminated silicon surface, on the other hand, the much smaller density of nucleation centers results in significantly larger islands and in a reduced density of grain boundaries. Note that the critical influence of the film morphology on the electrical characteristics of electron-doped pentacene films is also supported by recent experiments studying the conduction of rubidium-intercalated pentacene films deposited on glass. ${ }^{17}$ In that work, as-doped films exhibited an insulating temperature dependence of the conductivity. However, by performing a high-temperature annealing on the doped films, which results in an improved morphological quality, metallic behavior was also observed.

The sensitivity of the morphology of pentacene films to the substrate, together with the resulting effects on the elec- 

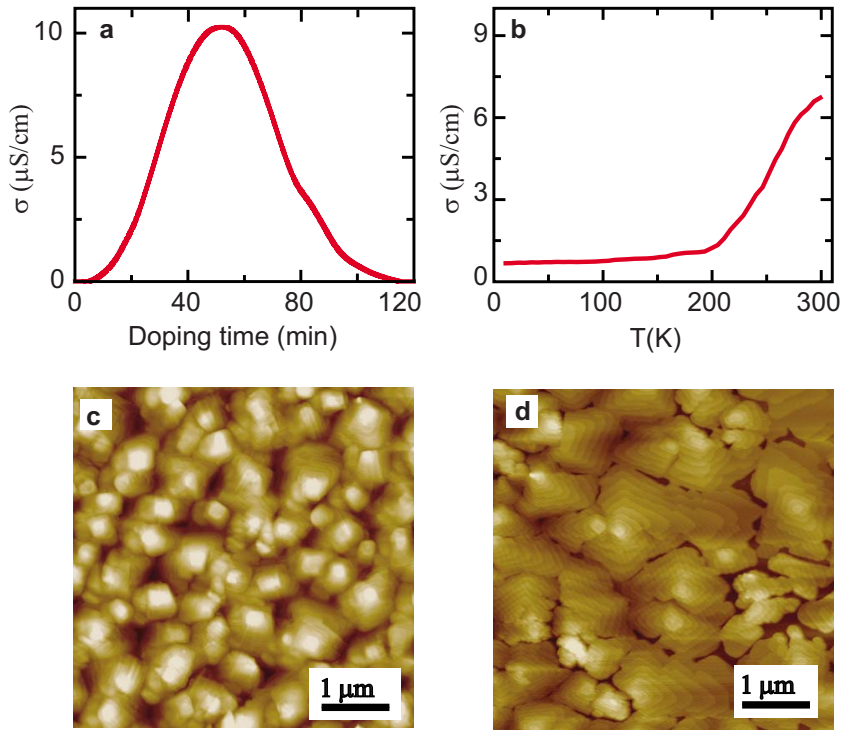

FIG. 3. (Color online) (a) Conductivity $\sigma$ measured at room temperature as a function of doping time for a 25 -nm-thick pentacene film deposited on $\mathrm{SiO}_{2}$. (b) Temperature dependence of the conductivity for a pentacene film grown on $\mathrm{SiO}_{2}$ and doped into the highest conductivity state. The conductivity is rapidly decreasing with lowering the temperature as it is typical for an insulator. [(c) and (d)] AFM images of pentacene films grown on $\mathrm{SiO}_{2}$ and on $\mathrm{H}$-terminated Si. (c) Small and randomly oriented grains with large height fluctuations are observed when the PEN films are deposited on $\mathrm{SiO}_{2}$, (d) whereas PEN films of similar thickness deposited on $\mathrm{Si}$ consist of large crystalline grains with a common relative orientation and only relatively small fluctuations in height.

tronic properties, is common to films of many conjugated molecules. In fact, a similar sensitivity was found in our earlier work on the electronic properties of alkali doped metal-phthalocyanine (MPc) films. ${ }^{19}$ Specifically, for films of $\mathrm{CuPc}$, NiPc, ZnPc, FePc, and MnPc, the maximum conductivity which can be achieved upon alkali doping when the films are deposited on $\mathrm{SiO}_{2}$ substrates is several orders of magnitude lower than the conductivity measured for films deposited on a Si surface and has always an insulating temperature dependence. Also for alkali doped $\mathrm{C}_{60}$ films, we observed that the surface termination of the substrate affects the morphology and the electronic transport properties of the
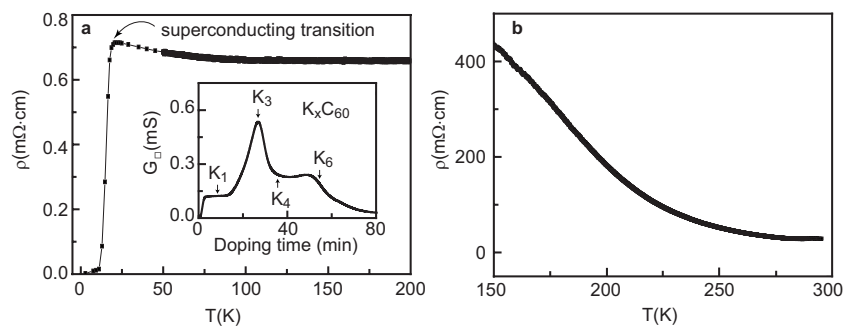

FIG. 4. (a) Temperature dependence of the resistivity of a highquality $\mathrm{K}_{3} \mathrm{C}_{60}$ film grown on $\mathrm{Si}$. As expected, the resistivity exhibits a superconducting transition at $18 \mathrm{~K}$. The inset shows the doping dependence of the conductivity. The conductance peak is typical of $\mathrm{K}_{3} \mathrm{C}_{60}$. (b) Temperature dependence of the resistivity of a $\mathrm{K}_{3} \mathrm{C}_{60}$ film grown on $\mathrm{SiO}_{2}$ showing insulating behavior. films. As illustrated in Fig. 4(a), the resistivity of $\mathrm{K}_{3} \mathrm{C}_{60}$ films grown on $\mathrm{Si}$ shows a low resistivity at low temperature and a transition to a superconducting state. On the contrary, the $\mathrm{K}_{3} \mathrm{C}_{60}$ films grown on $\mathrm{SiO}_{2}$ have significantly higher resistivity, exhibiting thermally activated transport [see Fig. 4(b)], without a superconducting transition.

\section{INTERPRETATION IN TERMS OF A MOTT STATE OF $K_{1}$ PEN}

The most striking aspect of our observations, namely, a sharp decrease in the conductivity starting at a carrier concentration of one electron per molecule concomitant with the re-entrance into an insulating state, has not been reported in earlier experiments on intercalated pentacene (in which the density of intercalants could not be determined ${ }^{14-18}$ ) or in studies of pentacene field-effect transistors with gate electrolytes (in which a metallic state has not been observed ${ }^{23}$ ). It implies that, contrary to the case of pentacene devices used in plastic electronics, the electronic properties of pentacene films at high carrier density cannot be described in terms of noninteracting electrons. In fact, even though it is known that pentacene molecules can accept only one electron and that doubly negatively charged pentacene ions do not exist ${ }^{24,25}$ (i.e., in our films charge transfer from the potassium atoms saturates at $1 \mathrm{~K} / \mathrm{PEN}$ ), a carrier concentration of one electron per molecule corresponds to a half-filled band and, for noninteracting electrons, should result in a metallic state. Therefore, interactions need to be invoked in order to explain our observations.

An established scenario for the formation of an insulating state at half-filling is the one of a Mott insulator emerging from strong electron-electron interactions. ${ }^{9,26}$ In a Mott insulator a strong Coulomb repulsion prevents two electrons to occupy the same pentacene molecule. Since at half-filling the motion of electrons necessarily requires double occupation of molecular sites, electron transport is suppressed and the system becomes insulating. This scenario is usually modeled theoretically using a Mott-Hubbard Hamiltonian, which includes a kinetic-energy term described within a tight-binding scheme and an on-site repulsion term. The Mott state occurs when this repulsion $(U)$ is larger than the bandwidth $(W)$ (determined by the tight-binding hopping amplitudes $t$ ). In this case the half-filled band splits into a lower (completely filled) and an upper (completely empty) Hubbard band, separated by a (Mott) gap of the order of $U$, when the interactions are strong. It is realistic that this scenario is realized in a molecular solid such as pentacene, in which the bandwidth is expected to be small owing to the absence of covalent bonds between the molecules.

To substantiate the Mott-insulator hypothesis we have analyzed the electronic structure using density-functional theory (DFT) calculations to extract the parameters of the Mott-Hubbard model. A main difficulty in doing this is that the structural knowledge of the intercalated films is incomplete, as our ultrahigh-vacuum setup is not equipped to perform in situ structural characterization, and ex situ characterization is impeded by oxidation of potassium when the sample is extracted from the vacuum system where the films 
are prepared. Therefore, for the DFT calculations we take advantage of the existing structural information on intercalated pentacene compounds and we determine the stable crystal structure of $\mathrm{K}_{1} \mathrm{PEN}$ using a computational relaxation procedure which refines the positions of all the atoms in the unit cell.

\section{A. Structural details of $K_{1}$ PEN}

It is well known from previous structural studies on pentacene films that the herringbone arrangement of the molecules is preserved when pentacene is intercalated with iodine $^{11-13}$ or with different alkali atoms ${ }^{14-18}$ and that intercalation takes place between the pentacene layers. It is also known that intercalation is accompanied by a considerable expansion of the unit-cell $c$ axis (by an amount close to the radii of the intercalated ions) while the in-plane lattice parameters $a$ and $b$ are only minorly affected. ${ }^{15,16}$

A reliable estimate of the length of the expanded $c$ axis is given by the sum of the radius of the alkali ion and the pristine $c$-axis parameter: the $c$-axis lattice constants that are obtained in this way for, e.g., RbPEN and CsPEN are within $2 \%$ of the experimental values. For $\mathrm{K}_{1} \mathrm{PEN}$ we construct the lattice parameters starting from two different polymorphs (one with $c=14.33 \AA$ and the other with $14.53 \AA$ ) using a $\mathrm{K}^{+}$ionic radius of $1.33 \AA$. The structures of the two polymorphs are taken from the experimental results in Ref. 27. They differ slightly in the packing of the pentacene molecules, which enables us to study the influence of realistic variations in the packing on the electronic structure. The precise length of the $c$ axis in the $\mathrm{K}_{1} \mathrm{PEN}$ is not critical for the resulting electronic structure. Our relaxation and bandstructure computations were checked for values up to $8 \%$ larger and smaller than the estimated $c$-axis parameters. We found that even such relatively large variations in $c$ do not affect our main results (i.e., the values of the calculated bandwidth $W$ and on-site repulsion $U$ ) because the interaction between adjacent pentacene layers is weak.

After constructing the unit cell of potassium-intercalated pentacene, using the information above to fix $a, b$, and $c$, we refine the positions of the atoms by a computational relaxation procedure. For all the electronic structure calculations we used the Vienna $a b$ initio simulation package (VASP) (Refs. 28 and 29) with projector augmented waves (PAWs) (Ref. 30) and the PW91 density functional. ${ }^{31}$ The selfconsistent calculations were carried out with an integration of the Brillouin zone using the Monckhorst-Pack scheme with a $6 \times 6 \times 4 k$-points grid and a smearing parameter of $0.01 \mathrm{eV}$ and a plane-waves basis set with a cutoff energy of $550 \mathrm{eV}$. To determine the stable structure of $\mathrm{K}_{1} \mathrm{PEN}$ all the atom positions in the unit cell are relaxed using a conjugategradient method. To avoid possible energy barriers we used a number of different initial configurations. In the relaxation procedure first the forces on the $\mathrm{K}$ ions are calculated and then the $\mathrm{K}$ positions are relaxed. We observe that the dopants move into high-symmetry positions in the plane between the pentacene layers. In the next step the positions of all atoms in the unit cell are relaxed-including the ones of the two PEN molecules. The final stable structure is the same for all different initial configurations. ${ }^{32}$
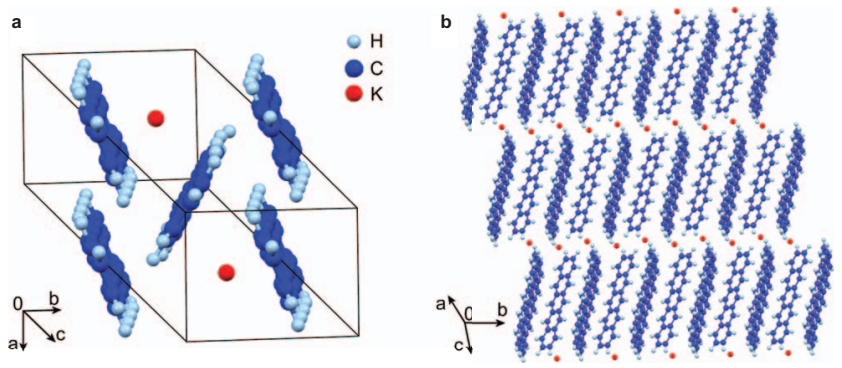

FIG. 5. (Color) Crystal structure of potassium-intercalated pentacene $\mathrm{K}_{1} \mathrm{PEN}$ obtained from $a b$ initio computational relaxation with (a) showing the herringbone of the PEN molecules and $\mathrm{K}$ atoms in the unit cell and (b) is a side view of the stacked layers of PEN and K, illustrating the potassium intercalation in between the molecular planes. The unit-cell parameters are $a, b, c$ $=6.239,7.636,15.682 \AA$ and $\alpha, \beta, \gamma=76.98^{\circ}, 88.14^{\circ}, 84.42^{\circ}$.

The optimized structure of $K_{1}$ PEN is shown in Fig. 5. We checked the reliability of the relaxation procedure on undoped pentacene and found that the calculated structure indeed corresponds to the actual known crystal structure of the material. In $\mathrm{K}_{1}$ PEN there are two inequivalent PEN molecules per unit cell, just as in the undoped compound. Intercalation changes the detailed molecular orientations in the unit cell (see Fig. 5), but we do not observe the formation of superstructures such as, for instance, molecular dimers. For the two distinct pentacene polymorphs ${ }^{27}$ for which we have performed the relaxation procedure, we found that the conclusions on electronic bandwidths and Coulomb interactions that will be presented hereafter hold equally well.

\section{B. Electronic structure and electronic correlations in $K_{1} P E N$}

Figure 6(a) shows the DFT band structure of $\mathrm{K}_{1} \mathrm{PEN}$ together with the projected density of states on the pentacene and potassium orbitals for the polymorph associated with the relaxed structure of Fig. 5. The Fermi energy lies in the middle of a half-filled band that is entirely of pentacene character, originating from its LUMO. The potassium derived electronic states are present only at much higher energy, demonstrating that little hybridization takes place and that the role of the potassium atoms is limited to transferring its electrons to the pentacene molecules. The total bandwidth is $W=0.7 \mathrm{eV}$. From a tight-binding fit of the band dispersion [see Fig. 6(b)] we extract the hopping amplitudes $t_{i j}$ that enter the kinetic-energy part of the Mott-Hubbard Hamiltonian

$$
H=\sum_{i} e_{i} n_{i}+\sum_{\langle i j\rangle, \sigma} t_{i j}\left(c_{i, \sigma}^{\dagger} c_{j, \sigma}+\text { H.c. }\right)+U \sum_{i} n_{i, \uparrow} n_{i, \downarrow},
$$

where we have two molecules in the unit cell with on-site energy $e_{i}$, the electron creation (annihilation) operators on site $i$ are $c_{i, \sigma}^{\dagger}\left(c_{i, \sigma}\right)$, with $\sigma$ as the electron spin, H.c. is the Hermitian conjugate, $n_{i, \sigma}=c_{i, \sigma}^{\dagger} c_{i, \sigma}, n_{i}=\Sigma_{\sigma} n_{i, \sigma}$, and $U$ is the effective Coulomb interaction between two electrons on the same molecule. The hopping integrals $t_{i j}$ are different in different directions and between nearest- and next-nearestneighbor molecules (see Table I). The resulting electronic 


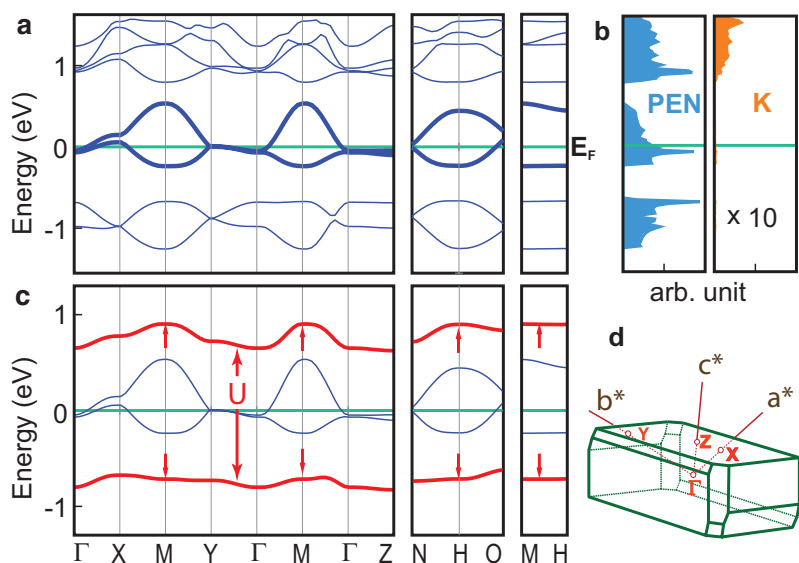

FIG. 6. (Color) Results of electronic structure calculations for $\mathrm{K}_{1} \mathrm{PEN}$. (a) Single-particle band structure, with the Fermi level $E_{F}$ (green line) as the zero of energy (Ref. 33). Valence and conduction bands are indicated by the thick blue lines. (b) Carbon (blue) and potassium (orange) projected density of states. (c) Tight-binding fit to the valence and conduction bands (blue thin lines) and the resulting lower and upper Hubbard bands from a mean-field analysis of the corresponding Hubbard Hamiltonian with $U=1.45 \mathrm{eV}$ (red thick lines). The arrows indicate the opening of the Hubbard gap. (d) Reciprocal lattice vectors and the first Brillouin zone of $\mathrm{K}_{1} \mathrm{PEN}$.

band structure [Fig. 6(a)] displays only very minor differences for the two stable polymorphs and within the present accuracy the tight-binding parameters are the same.

In order to determine the relative strength of electronic correlations and to compute the magnetic exchange interactions, the on-site Coulomb interaction $U_{\text {bare }}$ for two electrons on the same pentacene molecule is determined using the techniques described in Ref. 6. For this the total energy of neutral and charged pentacene molecules is calculated by density-functional calculations in the local-density approximation (LDA) using GAMESS with a double zeta plus polarization basis set (DZVP) basis set. ${ }^{34}$ The bare value of the Coulomb interaction is found to be $U_{\text {bare }}=3.50 \mathrm{eV}$. In the solid this value is screened, leading to a lower value $U .^{35-37}$ From the eigenvalues of the charged molecule that is placed inside a cavity of an homogeneous dielectric medium with dielectric constant of 3.3 using the surface and simulation of (volume) polarization for electrostatics $[\mathrm{SS}(\mathrm{V}) \mathrm{PE}]$ model,,${ }^{6,38}$

TABLE I. Tight-binding fit parameters to the $a b$ initio band structure of the half-filled conduction or valence band of KPEN. The on-site energy difference is denoted by $e$ and the hopping parameters along the $a, b$, and $c$ axes are denoted by $t$.

\begin{tabular}{lccccc}
\hline \hline Parameter & $\mathrm{meV}$ & Parameter & $\mathrm{meV}$ & Parameter & $\mathrm{meV}$ \\
\hline$e$ & 39 & & & & \\
$t_{a}$ & -33 & $t_{b}$ & -11 & $t_{c}$ & 1 \\
$t_{2 a}$ & -1 & $t_{a+b}$ & 1 & $t_{a-b}$ & -9 \\
$t_{a+c}$ & -6 & $t_{b+c}$ & 3 & $t_{a+b+c}$ & -5 \\
$t_{(a+b) / 2}$ & -96 & $t_{(a-b) / 2}$ & 90 & $t_{(3 a+b) / 2}$ & -4 \\
$t_{(3 a-b) / 2}$ & 9 & $t_{3 a / 2+b / 2+c}$ & 1 & $t_{a / 2+3 b / 2+c}$ & -3 \\
$t_{3 a / 2+3 b / 2+c}$ & -2 & $t_{3(a+b) / 2}$ & -3 & $t_{3(a-b) / 2}$ & 2 \\
\hline \hline
\end{tabular}

one finds $U=1.45 \mathrm{eV}$. We have also performed an independent estimate for the value of $U$ by considering the difference between the band gap of pristine pentacene from density-functional calculations $(0.7 \mathrm{eV})$ and its experimental value [2.2 eV (Ref. 39)], which gives $U \approx 1.5$. These two values, determined in two very different ways, are remarkably close. Very similar values for $U$ are found also for the second polymorph used in our calculations, indicating that these values are not very sensitive to differences in the structure. $^{40}$

From a straightforward self-consistent mean-field decoupling computation on the resulting Hubbard Hamiltonian the ground state is found to be a Néel ordered antiferromagnet, with a charge gap of $1.23 \mathrm{eV}$. The antiferromagnetic exchange between neighboring molecules in the plane is $J$ $=4 t_{(a \pm b) / 2}^{2} / U \simeq 290 \mathrm{~K}$. This value is actually an underestimation of the Heisenberg exchange, as certainly a nearestneighbor Coulomb interaction $V$ is also present, which has the effect of increasing the value of exchange by a factor of $U /(U-V) \cdot{ }^{41}$ We find that the coupling between molecules in neighboring planes, $J_{\perp}$, is 4 orders of magnitude lower than the in-plane $J$. Consequently $\mathrm{K}_{1} \mathrm{PEN}$ is a quasi-twodimensional antiferromagnet. Finally, an antiferromagnetic exchange of $\simeq 40 \mathrm{~K}$ is also present between in-plane nextnearest-neighbor molecules along the $a$ axis, leading to a weak frustration of the magnetic Néel ordering.

\section{DISCUSSION AND CONCLUSIONS}

Using the results of the electronic structure calculations we are now in a position to validate the Mott-state hypothesis. With a ratio $U / W \simeq 2.1$, electron-electron interactions cause the splitting of the LUMO band and the opening of a Mott gap, as shown in Fig. 6(c). The gap explains the observed re-entrance to the insulating state. Note that the Mottstate scenario also explains why the insulating state is only observed for a potassium concentration of 1.1-1.2 atoms per molecule (and not at exactly one [see Fig. 1(a)]). In fact, at exactly one potassium per pentacene molecule, nonuniformity in the potassium concentration-estimated to be approximately $10 \%-20 \%$ in our films-effectively dopes the Mott insulator causing the conductivity to remain large. However, even in the presence of nonuniformity, a potassium concentration slightly larger than $1 \mathrm{~K} / \mathrm{PEN}$ results in a uniform electron concentration exactly equal to one electron per molecule since, as we mentioned earlier, only one electron (and not two) can be donated to each pentacene molecule. $^{24,25}$ It should be noted that imperfections in the material, either due to disorder of the dopants or in the molecular arrangements, will lead to the presence of both disorder in the bandwidth and a local disorder potential. In general the physics of disordered Mott-Hubbard systems is very rich, ${ }^{42}$ but it is not a priori clear how relevant disorder will be in the present situation as we find that the Mott state in $\mathrm{K}_{1} \mathrm{PEN}$ is stabilized by a substantial electronic gap. The situation is similar for the detailed dependence of the transport properties on potassium concentration. The doping curves, Fig. 1 for instance, show a shoulder/peak in the conductivity at low density of unknown origin. It is clear on the other 
hand that in the presence of strong electron-electron interactions and impurity scattering the conductivity needs not be linear in carrier density. ${ }^{43}$

Since the coupling between pentacene molecules in different layers is very small and the electron-electron interaction is sufficiently large, the low-energy effective electronic Hamiltonian of the $\mathrm{K}_{x} \mathrm{PEN}$ reduces to the well-known twodimensional $t J$ model $^{9}$ with $t / J \approx 3-4$. Interestingly, the same $t J$ model in the same coupling regime describes another important class of materials, namely, strongly correlated cuprate superconductors such as $\mathrm{La}_{2-x} \mathrm{Sr}_{x} \mathrm{CuO}_{4}$. An apparent difference between these classes of materials is that in doped organics the formation of lattice polarons is expected to play a very important role.

We conclude that temperature-dependent transport measurements and theoretical calculations consistently indicate that at a doping concentration of one potassium ion per molecule potassium-intercalated pentacene is a strongly correlated Mott insulator, whose electronic properties are domi- nated by electron-electron interactions. An immediate consequence is the emergence of magnetism. Our calculations show that the magnetic interactions are dominated by a large positive magnetic exchange $J=4 t^{2} / U \simeq 290 \mathrm{~K}$ between electrons on nearest-neighbor molecules in the same pentacene layer. We predict that $\mathrm{K}_{1} \mathrm{PEN}$ is therefore an antiferromagnet. In fact, experimental indications for the presence of antiferromagnetism in intercalated pentacene have been reported in magnetic-susceptibility measurements performed in the past ${ }^{44}$ albeit at very low temperature.

\section{ACKNOWLEDGMENTS}

This work was supported by the Foundation for Fundamental Research on Matter (FOM), the Royal Dutch Academy of Sciences, the NWO Vernieuwingsimpuls, the NanoNed, and the Stichting Nationale Computerfaciliteiten. We are grateful to the FOM Institute for Atomic and Molecular Physics (AMOLF) for the RBS analysis of our samples.
${ }^{1}$ S. F. Nelson, Y.-Y. Lin, D. J. Gundlach, and T. N. Jackson, Appl. Phys. Lett. 72, 1854 (1998).

${ }^{2}$ C. D. Dimitrakopoulos and P. R. L. Malenfant, Adv. Mater. (Weinheim, Ger.) 14, 99 (2002).

${ }^{3}$ H. Sirringhaus, T. Kawase, R. H. Friend, T. Shimoda, M. Inbasekaran, W. Wu, and E. P. Woo, Science 290, 2123 (2000).

${ }^{4}$ G. H. Gelinck, H. E. A. Huitema, E. van Veenendaal, E. Cantatore, L. Schrijnemakers, J. B. P. H. van der Putten, T. C. T. Geuns, M. Beenhakkers, J. B. Giesbers, B.-H. Huisman, E. J. Meijer, E. M. Benito, F. J. Touwslager, A. W. Marsman, B. J. E. van Rens, and D. M. de Leeuw, Nature Mater. 3, 106 (2004).

${ }^{5}$ H. Klauk, U. Zschieschang, J. Pflaum, and M. Halik, Nature (London) 445, 745 (2007).

${ }^{6}$ G. Brocks, J. van den Brink, and A. F. Morpurgo, Phys. Rev. Lett. 93, 146405 (2004).

${ }^{7}$ T. Takenobu, T. Muro, Y. Iwasa, and T. Mitani, Phys. Rev. Lett. 85, 381 (2000).

${ }^{8}$ T. Ishiguro, K. Yamaji, and G. Saito, Organic Superconductors (Springer-Verlag, Berlin, 1998).

${ }^{9}$ M. Imada, A. Fujimori, and Y. Tokura, Rev. Mod. Phys. 70, 1039 (1998)

${ }^{10}$ L. Forro and L. Mihaly, Rep. Prog. Phys. 64, 649 (2001).

${ }^{11}$ T. Minakata, I. Nagoya, and M. Ozaki, J. Appl. Phys. 69, 7354 (1991).

${ }^{12}$ T. Minakata, H. Imai, and M. Ozaki, J. Appl. Phys. 72, 4178 (1992).

${ }^{13}$ T. Ito, T. Mitani, T. Takenobu, and Y. Iwasa, J. Phys. Chem. Solids 65, 609 (2004).

${ }^{14}$ T. Minakata, M. Ozaki, and H. Imai, J. Appl. Phys. 74, 1079 (1993).

${ }^{15}$ Y. Matsuo, S. Sasaki, and S. Ikehata, Phys. Lett. A 321, 62 (2004).

${ }^{16}$ Y. Matsuo, T. Suzuki, Y. Yokoi, and S. Ikehata, J. Phys. Chem. Solids 65, 619 (2004).

${ }^{17}$ Y. Kaneko, T. Suzuki, Y. Matsuo, and S. Ikehata, Synth. Met. 154, 177 (2005).
${ }^{18}$ B. Fang, H. Zhou, and I. Honma, Appl. Phys. Lett. 86, 261909 (2005).

${ }^{19}$ M. F. Craciun, S. Rogge, M. J. L. den Boer, S. Margadonna, K. Prassides, Y. Iwasa, and A. F. Morpurgo, Adv. Mater. (Weinheim, Ger.) 18, 320 (2006).

${ }^{20}$ R. W. I. de Boer, M. E. Gershenson, A. F. Morpurgo, and V. Podzorov, Phys. Status Solidi A 201, 1302 (2004).

${ }^{21}$ T. T. M. Palstra, R. C. Haddon, A. F. Hebard, and J. Zaanen, Phys. Rev. Lett. 68, 1054 (1992).

${ }^{22}$ R. Ruiz, B. Nickel, N. Koch, L. C. Feldman, R. F. Haglund, A. Kahn, and G. Scoles, Phys. Rev. B 67, 125406 (2003).

${ }^{23}$ M. J. Panzer and C. D. Frisbie, J. Am. Chem. Soc. 127, 6960 (2005).

${ }^{24}$ J. Szczepanski, C. Wehlburg, and M. Vala, Chem. Phys. Lett. 232, 221 (1995).

${ }^{25}$ T. M. Halasinski, D. M. Hudgins, F. Salama, L. J. Allamandola, and T. Bally, J. Phys. Chem. A 104, 7484 (2000).

${ }^{26}$ R. W. Lof, M. A. van Veenendaal, B. Koopmans, H. T. Jonkman, and G. A. Sawatzky, Phys. Rev. Lett. 68, 3924 (1992).

${ }^{27}$ C. C. Mattheus, A. B. Dros, J. Baas, A. Meetsma, J. L. de Boer, and T. T. M. Palstra, Acta Crystallogr., Sect. C: Cryst. Struct. Commun. 57, 939 (2001).

${ }^{28}$ G. Kresse and J. Hafner, Phys. Rev. B 47, 558(R) (1993).

${ }^{29}$ G. Kresse and J. Furthmüller, Phys. Rev. B 54, 11169 (1996).

${ }^{30}$ G. Kresse and D. Joubert, Phys. Rev. B 59, 1758 (1999).

${ }^{31}$ J. P. Perdew, J. A. Chevary, S. H. Vosko, K. A. Jackson, M. R. Pederson, D. J. Singh, and C. Fiolhais, Phys. Rev. B 46, 6671 (1992).

${ }^{32}$ The data file with the resulting structure for both polymorphs are available directly from the authors.

${ }^{33}$ The points in the Brillouin zone are $\Gamma=(0,0,0), X=\left(\frac{1}{2}, 0,0\right)$, $M=\left(\frac{1}{2}, \frac{1}{2}, 0\right), Y=\left(0, \frac{1}{2}, 0\right), Z=\left(0,0, \frac{1}{2}\right), N=\left(0, \frac{1}{2}, \frac{1}{2}\right), H=\left(\frac{1}{2}, \frac{1}{2}, \frac{1}{2}\right)$, and $O=\left(\frac{1}{2}, 0, \frac{1}{2}\right)$.

${ }^{34}$ M. W. Schmidt, K. K. Baldridge, J. A. Boatz, S. T. Elbert, M. S. Gordon, J. H. Jensen, S. Koseki, N. Matsunaga, K. A. Nguyen, S. Su, T. L. Windus, M. Dupuis, and J. A. Montgomery, Jr., J. 
Comput. Chem. 14, 1347 (1993).

${ }^{35}$ G. Giovannetti, G. Brocks, and J. van den Brink, Phys. Rev. B 77, 035133 (2008).

${ }^{36}$ J. van den Brink, M. B. J. Meinders, J. Lorenzana, R. Eder, and G. A. Sawatzky, Phys. Rev. Lett. 75, 4658 (1995); R. Eder, J. van den Brink, and G. A. Sawatzky, Phys. Rev. B 54, R732 (1996).

${ }^{37}$ M. B. J. Meinders, J. van den Brink, J. Lorenzana, and G. A. Sawatzky, Phys. Rev. B 52, 2484 (1995); J. van den Brink, R. Eder, and G. A. Sawatzky, Europhys. Lett. 37, 471 (1997).

${ }^{38}$ D. M. Chipman, J. Chem. Phys. 112, 5558 (2000).

${ }^{39}$ E. A. Silinsh, V. A. Kolesnikov, I. J. Muzikante, and D. R.
Balode, Phys. Status Solidi B 113, 379 (1982).

${ }^{40}$ In an alternative crystal structure for intercalated pentacene (see Ref. 45), a still smaller value of the bandwidth is found, implying an even larger correlation gap.

${ }^{41}$ R. Eder, J. van den Brink, and G. A. Sawatzky, Phys. Rev. B 54, R732 (1996).

${ }^{42}$ D. Belitz and T. R. Kirkpatrick, Rev. Mod. Phys. 66, 261 (1994).

${ }^{43}$ S. Fratini, H. Xie, I. N. Hulea, S. Ciuchi, and A. F. Morpurgo, New J. Phys. 10, 033031 (2008).

${ }^{44}$ T. Mori and S. Ikehata, J. Appl. Phys. 82, 5670 (1997).

${ }^{45}$ A. Hansson, J. Bohlin, and S. Stafstrom, Phys. Rev. B 73, 184114 (2006). 